\title{
9. \\ Einige Bemerkungen über elliptische Functionen.
}

(Vom Herrn Prof. Dr. Gudermann za Münster.)

1. Eine durch merkwürdige Eigenschaften ausgezeichnete sphärische transcendente Curve, deren Gleichung

$$
\cos \left(k^{\prime} \cdot x\right) \cdot \cos y=k^{\prime}
$$

ist, wo $x$ die Abscisse und $y$ die zugehörige senkrechte Applicate eines beliebigen Punctes der Curve, $k=\sin \theta$ den Modul, also $k^{\prime}=\cos \theta$ das Complement des Moduls vorstellt, versinnlicht am besten den Zusammenhang aller elliptischen Functionen und Amplituden mit dem Argumente. Jene Gleichung hat Ähnlichkeit mit der Gleichung $\cos x \cdot \cos y=k^{\prime}$ eines sphärischen Kreises, dessen Radius $\theta$ ist, und die Curve selbst stimmt in vielen Eigenschaften mit dem Kreise überein. Setzt man $\theta=\frac{\pi}{2}$, so verwandelt sich die obige Gleichung, in welcher nun aber der Anfangspunct verlegt werden mufy, in

$$
\cos y=\frac{1}{1+x}
$$

d. b. in die Gleichung der sphärischen Longitudinale, welche ebenfalls mebrere bemerkenswerthe Eigenschaften besitzt.

2. Der Zusammenhang zwischen den elliptisohen Functionen, ihren Amplituden und dem Argumente wird auch geometrisch dargestellt durch eine sphärische Curve, deren Gleichung

$$
\rho=\operatorname{am}(s) \text { und } v=k . s
$$

ist, wo $\varrho$ einen sphïrischen Leitstrahl eines Winkels, $v$ den verïnderlichen Winkel, welchen der Leitstrahl mit seiner ursprünglichen Richtung macht, und $s$ den Bogen der Curve vorstellt. Der Winkel, welchen eine Berührungslinie der Curve mit dem L’eitstrahle $\rho$ dex Berührungspunctes macht, ist dann $=\operatorname{am}\left(k s, \frac{1}{k}\right)$, und durch eben diese Amplitude kann auch die Flïche ausgedrückt werden. Die Curve lïuft, in unzähligen Windungen um die Kugel, unendliche Male durch den Punct, von welchem aus die 
Leitstrahle gezogen werden, und durch seinen Gegenpunct gehend, aber immer in anderen Richtungen, und läuft also nur in speciellen Filleon in sich zurück.

3. Die Gleichung

$$
\cos y \cdot \operatorname{Sin}\left(\frac{k^{\prime}}{k} x\right)=\frac{k^{\prime}}{k}
$$

in welcher $x, y, k$ und $k^{\prime}$ die obige Bedeutung haben, stellt ebonfalls eine interessante Curve dar; die Applicate $y$ ist jetzt die Amplitude des zugehörigen Bogens der Curve für den Modul $k$ (oder $y=\operatorname{am}(s)$ ), und der Winkel, welchen ein berührender Hauptkreis mit der Applicate des Berührungspunctes macht, ist wieder $=\operatorname{am}\left(k s, \frac{1}{k}\right)$, nach der in denSchriften des Herrn Prof. Jacobi gängigen Bezeicbnung.

Münster, im Januar 1836. 